\title{
USO DE TRAMPAS CON FEROMONA EN EL MUESTREO DEL PEQUEÑO BARRENADOR DEL FRIJOL, Elasmopalpus lignosellus (Zeller) (Lepidoptera: Pyralidae) ${ }^{1}$
}

\author{
Jesús Loera ${ }^{2}$, Robert Lynch ${ }^{3}$, Raúl Rodríguez ${ }^{2}$
}

\begin{abstract}
RESUMEN
Uso de trampas con feromona en el muestreo del pequeño barrenador del frijol, Elasmopalpus lignosellus (ZELLER) (Lepidoptera: Pyralidae). Trampas con feromona fueron usadas para adultos de pequeño barrenador, Elasmopalpus lignosellus (Zeller), en frijol común Phaseolus vulgaris L., en Río Bravo Tamaulipas, México. Trampas Pherocon 1C con 10 microgramos de feromona de pequeño barrenador fueron más efectivas cuando se colocaron a una altura de $0,5 \mathrm{~m}$ arriba del nivel del suelo. La ubicación de las trampas: en las orillas o dentro de la parcela, a lo largo de los surcos o transversal a los surcos capturaron cantidades similares de adultos de pequeño barrenador. Trampas de "hielo seco" fueron más efectivas que las trampas Pherocon $1 \mathrm{C}$ a $0,5 \mathrm{~m}$. arriba del nivel del suelo. Estos dos tipos de trampas capturaron cantidades similares de adultos cuando fueron colocadas a alturas de 1,0, 1,5 y 2,0 m. arriba del nivel del suelo. Se discute la factibilidad del uso de estas trampas para adultos de pequeño barrenador durante todo el desarrollo del cultivo del frijol.
\end{abstract}

\begin{abstract}
Lesser cornstalk borer monitoring with the use of pheromone traps, Elasmopalpus lignosellus (ZELLER) (Lepidoptera: Pyralidae). Pheromone traps were used to monitor adult populations of the lesser cornstalk borer (Elasmopalpus lignosellus (Zeller) in beans (Phaseolus vulgaris L.) near Rio Bravo, Tamaulipas, Mexico. Pherocon 1C traps baited with 10 micrograms of lesser cornstalk borer pheromone in rubber septa were mast effective when placed at $0.5 \mathrm{~m}$ high above the ground. Traps setting in the field, along the border of the field, within the row, or across rows produced comparable captures of adult males. Pie plate sticky traps were more effective than pherocon $1 \mathrm{C}$ traps at $0.5 \mathrm{~m}$. These two types of traps captured similar quantities of males at 1.0,1.5, and $2.0 \mathrm{~m}$ heights. The feasibility of using these traps for monitoring' lesser cornstalk borer adults along the bean growing season is discussed.
\end{abstract}

\section{INTRODUCCION}

El pequeño barrenador (PB), Elasmopalpus lignosellus (Zeller), es un insecto polífago, común en leguminosas y gramíneas, y es una plaga importante en el sur de Estados Unidos y otras áreas subtropicales (Luginbill y Ainslie 1917, Tippins 1982). En México, sin embargo, el PB es relativamente desconocido y es rara vez observado atacando cultivos agrícolas. En el Noreste de México, el PB ha causado ocasionalmente daño económico en plántulas de maíz, Zea mays (L.) al reducir la densidad de po- blación de plantas y subsecuentemente el rendimiento (Loera 1983). No obstante, cuando el frijol común, Phaseolus vulgaris L., se siembra en superficies considerables de hasta 100,000 hectáreas en el noreste de México, las infestaciones del PB son comunes y se desarrollan tan rápidamente que para cuando las larvas son detectadas el daño ya ha sobrepasado el nivel económico.

El comportamiento del PB hace difícil la detección de infestaciones antes del daño económico. La mayoría de los procedimientos de muestreo involucran prácticas

\footnotetext{
1 Presentado en la XL Reunión Anual del PCCMCA en Costa Rica, América Central. 13 al 19 de marzo, 1994.

2 INIFAP-CIRNE Apdo 172 Rio Bravo Tam. Mexico.

3 USDA-ARS Tifton, GA 31793-0748.
} 
consumidoras de tiempo y esfuerzo al revisar plantas y suelo (Womack et al. 1981).

Jones y Bass (1979) evaluaron trampas en el suelo para muestrear larvas de PB y concluyeron que esta técnica capturó larvas grandes siendo poco eficiente en la captura de larvas pequeñas.

Para insectos como el PB, difíciles de muestrear en sus estados larvales, la captura de adultos en trampas con feromona representa un medio para estimar la densidad de la población inicial. La simple detección de la ocurrencia inicial de adultos es importante si se requiere aplicar medidas de control preventivas o bien para determinar si un muestreo mas intensivo de los estados dañinos del PB es necesario. Lynch et al. (1984) identificaron y evaluaron la feromona sexual del PB que puede ser usada para determinar sus poblaciones. El estudio actual fue conducido para determinar el potencial de las trampas con feromona para muestrear de las poblaciones de adultos del pequeño barrenador en frijol.

\section{MATERIALES Y MÉTODOS}

El ensayo se desarrolló en Río Bravo, Tamaulipas, México, de septiembre a diciembre de 1992 y se utilizaron tres parcelas comerciales de frijol, de 10 hectáreas cada una.

Se evaluaron la eficiencia de la altura y ubicación de las trampas con feromona para capturar adultos de P. El arreglo de los tratamientos se hizo en parcelas divididas bajo un diseño de bloques completamente al azar con tres repeticiones: Las parcelas grandes fueron las alturas de las trampas: 0,5, 1,0, 1,5, y 2,0 m árriba del nivel del suelo (a.n.s.) y las subparcelas fueron la ubicación de las trampas: a lo largo de un surco central de la parcela; transversal a los surcos en la parte central de la parcela; a lo largo del surco orillero de la parcela, y transversal a los surcos en una orilla de la parcela.

Las trampas utilizadas fueron del tipo pherocon $1 \mathrm{C}$ descritas por Trece Inc. (1987) y estuvieron separadas por $5 \mathrm{~m}$ una de otra. La feromona de PB consistió de 36,8\%
(Z)-7-tetradecen-1-ol acetato, 19,7\% (Z)-9-tetradecen-1ol acetato, 38,8\% (Z)-11-hexadecen-1-ol acetato, y 4,7\% (Z)-9-tetradecen-1-ol. Una porción de caucho conteniendo 10 microgramos de feromona sintética fue colocado en el centro de la parte inferior de cada trampa. Las caras internas de las pactes superior e inferior de las trampas estuvo impregnado con un pegamento inalterable a diversas temperaturas. La feromona fue reemplazada cada diez días y los adultos de PB capturados fueron removidos en cada revisión. Se registró el número de machos adultos capturados cada tercer día.

Un segundo experimento fue diseñado para comparar las trampas Pherocon $\mathrm{IC}$ contra trampas elaboradas manualmente con base a dos platos de "hielo seco" de las descritas por Sparks et al. (1979). Los tratamientos fueron arreglados en parcelas divididas bajo un diseño de bloques completamente al azar con cuatro repeticiones y fue conducido en una parcela comercial de diez hectáreas de frijol. Las trampas estuvieron separadas por $5 \mathrm{~m}$ una de otra. Las parcelas grandes fueron los dos tipos de trampas con feromona ej. Pherocon $1 \mathrm{C}$ vs. platos de "hielo seco". Las subparcelas fueron las alturas de las trampas ej. 0,5, 1,0, 1,5 y 2,0 m. Las subparcelas fueron las ubicaciones de las trampas de manera similar a como se mencionó en el experimento previo. Así mismo, la cantidad de feromona por trampa, el reemplazo de la feromona, la remoción de adultos capturados y los datos registrados se hicieron de la misma manera descrita anteriormente.

\section{RESULTADOS Y DISCUSIÓN}

Las densidades de población de PB variaron grandemente entre parcelas (repeticiones). Considerablemente más adultos fueron capturados en la parcela 1 que en las otras dos parcelas. En la parcela tres se capturaron el doble de adultos que los capturados en la parcela dos (Cuadro 1 ). Estas diferencias pueden deberse a la variación del tipo de suelo entre parcelas o a la variación en el estado de desarrollo del frijol entre parcelas, dado que, tanto el tipo de suelo como el estado de desarrollo de las plantas, son factores determinantes que influyen en la infestación de PB (Tippins 1982). 
Cuadro 1. Número promedio de adultos de pequeño barrenador capturados en trampas con feromona Pherocon 1C de diferente altura y en diferente parcela de frijol.

\begin{tabular}{lccc}
\hline $\begin{array}{l}\text { Altura de } \\
\text { trampa }(\mathbf{m} .)\end{array}$ & $\begin{array}{c}\text { No. de adultos } \\
\text { capturados/trampa }\end{array}$ & $\begin{array}{c}\text { Parcela } \\
\text { capturados/trampa }\end{array}$ & No. de adultos \\
\hline 0,5 & $6,62 \mathrm{a}$ & 1 & 24,96 \\
1,0 & $1,99 \mathrm{~b}$ & 2 & 5,03 \\
1,5 & $1,10 \mathrm{c}$ & 3 & 10,56 \\
2,0 & $0,45 \mathrm{c}$ & & \\
\hline
\end{tabular}

Números seguidos por diferente letra son significativamente diferentes al nivel $\mathrm{P}<0,05$ usando la prueba de rango múltiple de Duncan.

Comparación del número promedio de adultos de PB capturados a diferentes alturas de trampas también mostró diferencias altamente significativas. Significativamente más $(\mathrm{P}<0,05)$ adultos fueron capturados en trampas 0,5 m. que en trampas $1,0,1,5$, ó 2,0 m. (a.n.s.). De manera similar, significativamente más adultos fueron capturados en trampas 1,0 m. que en trampas 1,5 ó 2,0 m. a.n.s. Lynch et al. (1984) reportaron una variación en la respuesta de PB capturados en trampas desde 0,3 a $1,2 \mathrm{~m}$. a.n.s. en parcelas de cacahuate. Sin embargo indicaron también que el mayor número de machos fueron capturados en trampas puestas a 0,9 m. a.n.s. Los resultados del presente estudio muestran que trampas $0,5 \mathrm{~m}$ (a.n.s.) fueron significativamente más eficientes que trampas $1,0 \mathrm{~m}$ (a.n.s.) en la captura de machos adultos de PB. A pesar de que las plantas de frijol y cacahuate son aproximadamente de la misma altura, la altura de la trampa es mucho más importante para monitorear poblaciones de PB en frijol que en cacahuate.

La ubicación de las trampas tuvo poca influencia en el número de machos capturados en las trampas con feromona. No hubo diferencias significantes $(\mathrm{P}<0,05)$ en capturas cuando las trampas estuvieron ubicadas ya sea a lo largo o transversal a los surcos o cuando las trampas estuvieron ubicadas en la orilla o en la parte central de la parcela. Estos resultados de capturas de PB en frijol difieren de las capturas de PB en cacahuate reportadas por Lynch et al. (1984). Ellos indican haber capturado más adultos en las trampas ubicadas en la parte central de parcelas de cacahuate que en las trampas ubicadas en las orillas.

La interacción entre altura y ubicación de trampas no fue significativa. Las trampas de una altura dada fueron igualmente efectivas en capturar adultos de PB cuando estuvieron ubicadas en la orilla o en la parte central de la parcela de frijol, así mismo a lo largo de los surcos como transversal a los surcos.

La Figura 1 muestra una comparación del número de machos de PB capturados por trampa para las cuatro alturas de trampa durante setiembre-octubre. Las trampas 0,5 m. a.n.s. fueron mucho más efectivas que las trampas 1,0 , 1,5 y 2,0 m. a.n.s. Comparación de trampas Pherocon 1C contra trampas de "hielo seco" mostróque estas últimas fueron significativamente $(\mathrm{P}<0,05)$ más efectivas para capturar adultos de PB (Cuadro 2). La interacción de tipo de trampa y altura de trampa fue significante. Análisis de la interacción indicó que las trampas de "hielo seco" capturaron significativamente más $(\mathrm{P}<0,05)$ machos de $\mathrm{PB}$ que las trampas Pherocon $1 \mathrm{C}$ a $0,5 \mathrm{~m}$. a.n.s., pero los dos tipos de trampa fueron igualmente efectivas a 1,0, 1,5 y 2,0 m. a.n.s. La ubicación de las trampas en la orilla o en la parte central de la parcela o bien a lo largo o transversal 


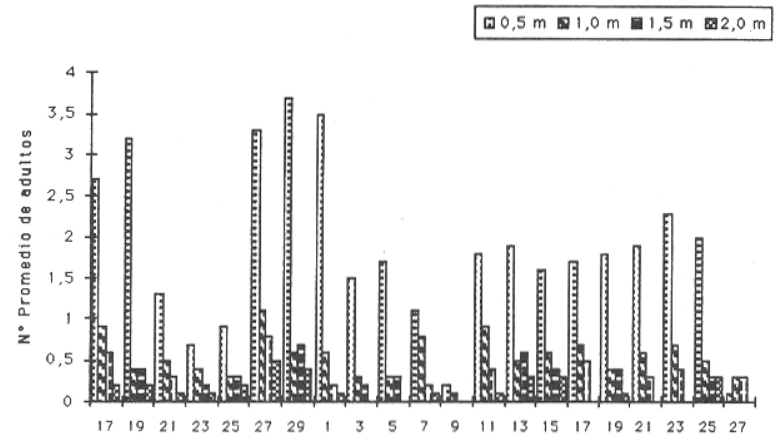

Fig. 1. Promedio de adultos de pequeño barrenador en trampas Pherocon 1C a cuatro alturas. Septiembre-octubre, 1992.

Cuadro 2. Número promedio de adultos de pequeño barrenador capturados en trampas con feromona Pherocon 1C y de "hielo seco" a diferentes alturas.

\begin{tabular}{ccc}
\hline $\begin{array}{c}\text { Altura de } \\
\text { trampa (m.) }\end{array}$ & $\begin{array}{c}\text { Trampas } \\
\text { Pherocon 1C }\end{array}$ & $\begin{array}{c}\text { Trampas } \\
\text { "hielo seco" }\end{array}$ \\
\hline 0,5 & $1,50 \mathrm{~b}$ & $3,39 \mathrm{a}$ \\
1,0 & $1,23 \mathrm{bc}$ & $0,73 \mathrm{~cd}$ \\
1,5 & $0,17 \mathrm{~d}$ & $0,33 \mathrm{~d}$ \\
2,0 & $0,00 \mathrm{~d}$ & $0,22 \mathrm{~d}$ \\
\hline
\end{tabular}

Números seguidos por diferentes letras son significativamente diferentes al nivel $\mathrm{P}<0,05$ usando la prueba de rango múltiple de Duncan.

a los surcos produjo resultados no significativos $(\mathrm{P}<0,05)$ como en el experimento anterior.

Las Figuras 2, 3, 4 y 5 presentan una comparación de las capturas en las trampas Pherocon 1C y trampas de "hielo seco" a diferentes alturas.

Las trampas de "hielo seco" capturaron más adultos de PB que las trampas Pherocon $1 \mathrm{C}$ en todas las fechas cuando su altura fue de 0,5 m a.n.s. Sin embargo, los dos tipos de trampa capturaron considerablemente muy pocos adultos cuando estuvieron a 1,0, 1,5 y 2,0 m. a.n.s., y los resultados variaron grandemente entre los dos tipos de trampa.

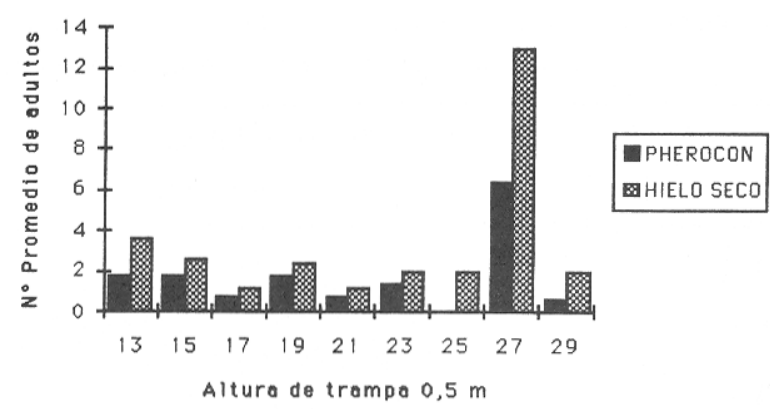

Fig. 2. Promedio de adultos de pequeño barrenador capturados en trampas Pherocon $1 \mathrm{C}$ o de Hielo Seco. Noviembre, 1992.

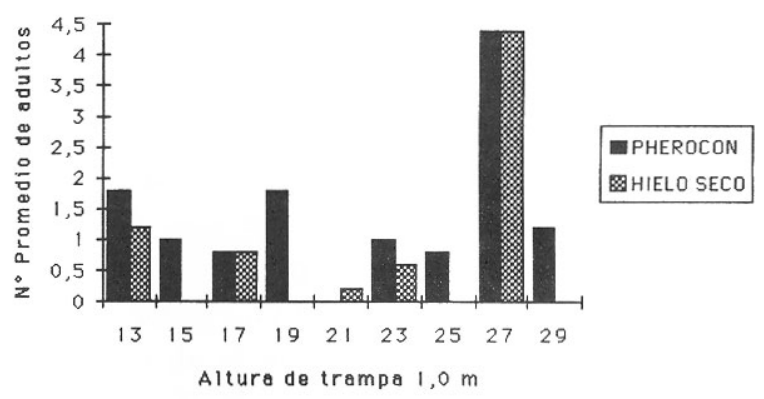

Fig. 3. Promedio de adultos de pequeño barrenador capturados en trampas Pherocon 1C o de Hielo Seco. Noviembre, 1992.

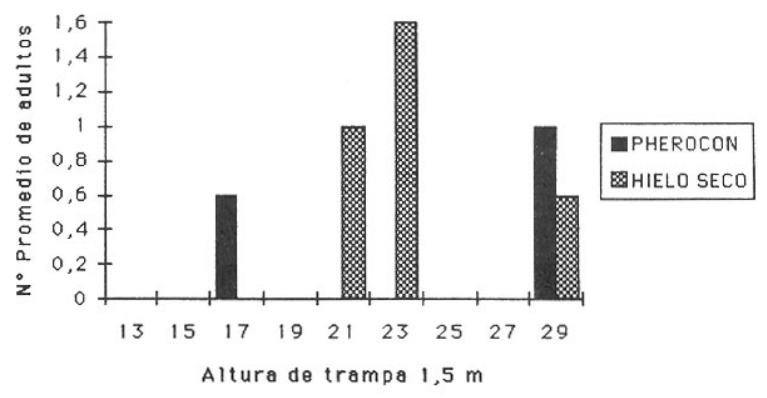

Fig. 4. Promedio de adultos de pequeño barrenador capturados en trampas Pherocon 1C o de Hielo Seco. Noviembre, 1992. 


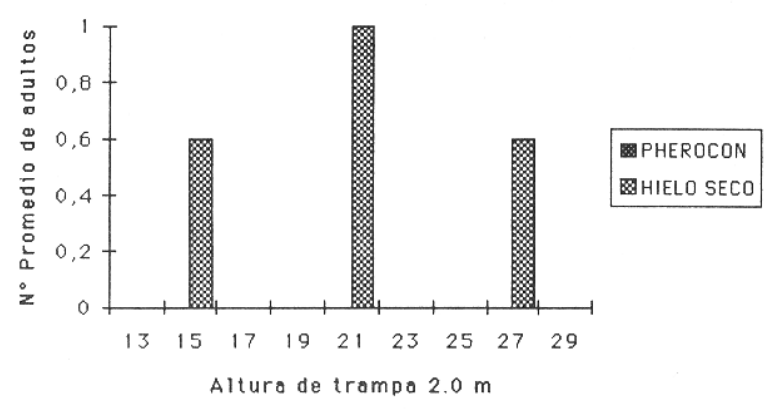

Fig. 5. Promedio de adultos de pequeño barrenador capturados en trampas Pherocon 1C o de Hielo Seco. Noviembre, 1992.

\section{CONCLUSIONES}

Las trampas con feromona sirven para detectar las densidades iniciales de la población de adultos, de insectos difíciles de muestrear en su estado larval como el PB. En el presente estudio se lograron capturar los adultos de PB responsables de la infestación inicial en frijol común.

Las trampas Pherocon 1C al igual que las trampas "hielo seco" cada una con 10 microgramos de feromona de PB, fueron más eficientes para capturar adultos del PB en frijol cuando se instalaron a una altura de $0,5 \mathrm{~m}$. a.n.s. en comparación con alturas de 1,0, 1,5 ó 2,0 m. a.n.s.

Las trampas de "hielo seco" fueron más eficientes que las trampas Pherocon 1C a 0,5 m. a.n.s. para capturar adultos del PB. Esto permite mayor accesibilidad a las trampas ya que pueden ser construidas con materiales fáciles de conseguir en cada localidad.

La ubicación de las trampas en los bordes o dentro de la parcela de frijol ya sea en dirección de los surcos o transversal a ellos, tuvo poco efecto en la captura de adultos de PB.

Para capturar los primeros adultos de PB que infestan al Frijol común, se sugiere el empleo de trampas de "hielo seco" a una altura de $0,5 \mathrm{~m}$, (a.n.s.) a lo largo de los surcos ya que de esta manera no se interrumpirá el movi- miento de la maquinaria dentro de la parcela y las trampas podrán ser mantenidas en el mismo lugar durante todo el ciclo del cultivo para el seguimiento de poblaciones de adultos de PB, ya que el daño ocurre desde la plántula hasta la formación de vainas.

\section{LITERATURA CITADA}

JONES, D.; BASS, M. H. 1979. Evaluation of pitfall traps for sampling les ser cornstalk borer larvae in peanuts. J.Econ. Entomol. 72:289-290.

LOERA, G. J. 1983. Insectos dañinos del maíz. Manual Fitosanitario Regional PIFSY-SARH Deleg. Zona Norte Tamaulipas, Mex. p.73-103.

LUGINBILL, P.; AINSLIE, G. 1917. The lesser corn-stalk borer. U.S. Dep. Agric. Bul!. No. 539, 27p.

LYNCH, R. E.; KLUN, J. A.: LEONHARDT. B. A.: SCHWARZ, M.; GARNER, J. W. 1984. Female sex pherornone of the les ser cornstalk borer, Elasmopalpus lignosellus (Lepidoptera: Pyralidae). Environ. Entomol. 13:121-126.

SPARKS, A. N.; CARPENTER, J. E.; MULLINIX, B. G. 1979 Field res pon se of male Heliothis zea (Boddie) to pheromonal stimuli and trap design. J. Ga. Entomol. Soc. $14: 318-325$

TIPPINS, H. H. 1982. A review of information on the lesser cornstalk borer Elasmopalpus lignosellus (Zeller). Ga. Agric. Exp. Stn. Spec. Publ. No. 17, 65 p.

TRECE INC. 1987. Goodbye guess work. Bulletin of the Ent. Soc. of Am. Fal!. 1987. p. 133.

WOMACK , H.; FRENCH, J. C.; 1OHNSON, F. A.; THOMPSON, S. S.; SWANN, C. W. 1981. Peanut pest management in the southeast. Ga. Coop. Ext. Serv. Bull. 850,26 p. 